\title{
Surface roughness influence on photothermal radiometry
}

by H. G. Walther

Universitaet Jena, Institut fuer Optik und Quantenelektronik, Max-Wien-Platz 1, D-07743 Jena, Germany,

E-mail: walther@ioq.uni-jena.de

\section{Abstract}

Surface roughness has to be taken into account for accurate interpretation of photothermal measurements. An expression for photothermal signals from rough samples was derived by combining the effect of roughness induced thermal wave dispersion with the equivalent layer model. The performance of the proposed model was successfully demonstrated by comparing calculations with radiometric measurements from rough steel samples.

\section{Introduction}

Industrial photothermal techniques are often applied to specimens with rough surfaces. For accurate data interpretation it is necessary to understand the role that the surface texture plays at photothermal signal generation. An example for roughness influenced radiometric signals is shown in Fig.1 [1]. The measured phase curves depend characteristically on surface roughness. Interpreting that fact the roughness influence on photothermal signals can be mathematically eliminated. This was proposed and successfully demonstrated in [2]

In the literature surface roughness affected photothermal signals were considered since about 1985 [3]. Bein and coworkers interpreted the observed frequency scans by an effective surface layer with diminished thermal effusivity. The roughness induced modification of thermal transport properties was modeled by appropriate assumptions about geometry and/or statistics of the surface (see for instance [4]). Another approach was fractal description of rough surfaces (Boccara et.al. [5] and Osiander et.al. [6]). A rigorous description solution of the heat diffusion equation in the vicinity of rough boundaries was presented by D.Vandembroucq and Roux [7]. They used conformal mapping technique to stretch the rough boundary by a tailored coordinate transformation. The same transformation was applied also to heat diffusion equation and boundary conditions. For constant heat flux the temperature field was calculated and conclusions about the roughness induced distortion of isotherms were drawn.

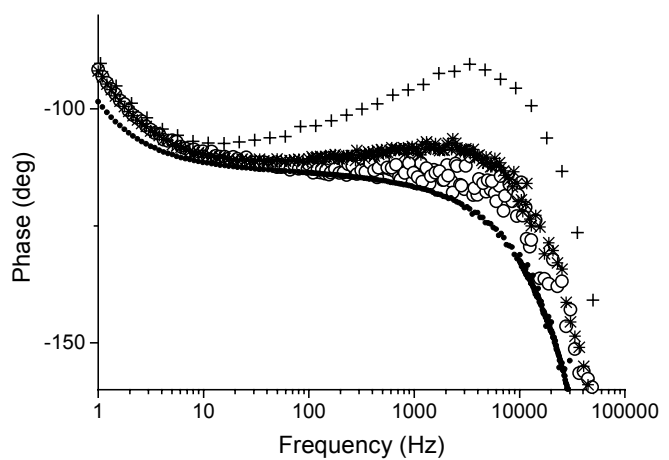

Fig.1. Radiometric phase measurements from rough steel samples and polished zirconia used as reference (• reference, o polished steel, * slightly rough, + very rough) 
The aim of this paper is to find out how photothermal signals depend on surface texture parameters. For this reason we studied theoretically the relation between roughness parameters and effective thermal transport properties. We made use of the constant heat flux results obtained in [7] and generalized them for thermal wave propagation. The found relationship was finally tested by comparing calculations with radiometric measurements from rough specimens.

\section{Mathematical description of rough surfaces}

Roughness describes disordered deviations of the surface under study from the ideal smooth plane. Roughness profiles can be quantified in different ways:

A) In the industrial practice, surface texture parameters are defined in standards and are estimated commonly by optical or stylus profilometry. Essential numbers of technical importance are: i) Rp is maximum profile deviation from mean plane (2.Rp is the so-called peak-to-valley distance); ii) $\mathrm{Rq}$ denotes random mean squared height deviation which is defined by $R q=\sqrt{\frac{1}{L} \cdot \int_{0}^{L}\left|z^{2}(x)\right| \cdot d x} . \mathrm{L}$ is the distance along which the height deviations $\mathrm{Z}(\mathrm{X})$ are averaged; iii) $\mathrm{RSm}$ is the mean distance between dominant grooves. It can be interpreted as the maximum spatial wavelength of the rough profile.

B) From a more mathematical point of view, the statistical approach to roughness parameters is quite instructive. A stochastically fluctuating surface profile $Z(X)$ can be characterized by its spatial frequency content which is expressed by the power spectral density (PSD) $g(k)$. With Fourier transform $Z(k)=F(Z(X))$ of the profile $Z(X)$ it is $g(k)=|F(Z(X))|^{2}$. For technical applications two different types of surface texture have to be distinguished: waviness and roughness. Both effects can be separated by appropriate kfrequency-filtering of the profile with characteristic cut-off k-frequencies. In this paper we restrict our consideration to roughness. The random mean squared roughness $\mathrm{s}$ is related to $\mathrm{g}(\mathrm{k})$ by

$$
s^{2}=2 \cdot \int_{k_{\min }}^{k_{\max }} g(x) \cdot d k
$$

C) Many surfaces can be appropriately characterized by self-affine profiles obeying the scaling rule within the spatial frequency range bound by $k_{\min }$ and $k_{\max }$

$$
x \rightarrow b \cdot x \text { and } z \rightarrow b^{\varsigma} z
$$

$\mathrm{b}$ is a lateral stretching factor. The so-called roughness exponent $\zeta(0<\zeta<1)$ controls the vertical structure of profile and estimates the power law of PSD according to

$$
g(x)=\frac{1}{k^{1+2 \varsigma}}
$$

\section{Calculation of the frequency dependent photothermal signal}

As shown earlier ([2-4]) the measured photothermal signals can be described by an effective layer on substrate. The equivalency between rough half-space and effective layer

Sample is sketched in Fig.2. Nonstationary heat transport in the bulk is controlled by thermal diffusivity $\alpha$, and thermal effusivity $\varepsilon$, which are connected with thermal conductivity $K$, mass density $\rho$ and specific heat $c$ by the relations $\alpha=k / \rho \cdot c$ and $\varepsilon=(k \cdot \rho \cdot c)^{1 / 2}$. Obviously, the thermal properties of the effective layer differ from the bulk. They are marked by primes (') to distinguish them from bulk properties. The layer thickness $d$ is assumed to be of the order of the maximum distance between profile peaks and valleys. 
For the ratio $S$ of the surface temperature $T$ ' of the effective layer-on-substrate system and the smooth-half-space temperature $T_{\text {PLANE }}$ we got the expression [4]

$$
S=\frac{T^{\prime}}{T_{\text {PLANE }}}=\frac{\varepsilon}{\varepsilon^{\prime}} \cdot \frac{1+R(f) \cdot \exp \left(-2 \sigma^{\prime} d\right)}{1-R(f) \cdot \exp \left(-2 \sigma^{\prime} d\right)}
$$

with thermal reflection coefficient $R(f)=\frac{\varepsilon^{\prime}(f)-\varepsilon}{\varepsilon^{\prime}(f)+\varepsilon}$ and thermal wave number

$$
\sigma^{\prime}(f)=\sqrt{i \cdot 2 \pi f / \alpha^{\prime}} .
$$

To find $\varepsilon^{\prime}$ and $\alpha^{\prime}$ we make use of the following result from [7]: comparing the distances between mean surfaces and corresponding isotherms there is a difference between plane and rough surfaces. In the rough case that distance is shorter than for the plane case by length $\mathrm{H}$

$$
\langle H\rangle=C(\varsigma) \cdot k_{\min } \cdot\left\langle s^{2}\right\rangle
$$

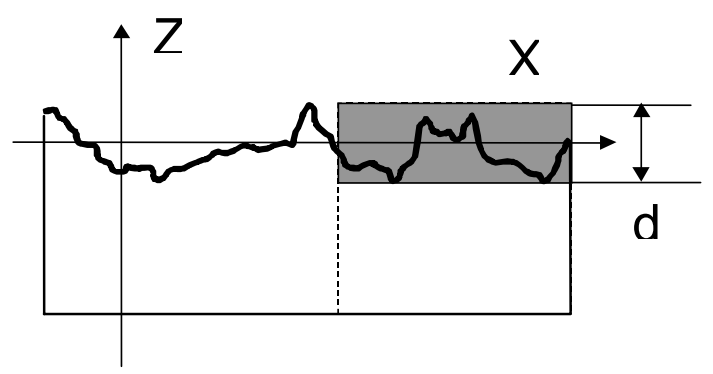

Fig. 2. Thermal equivalence between rough surface and effective layer model

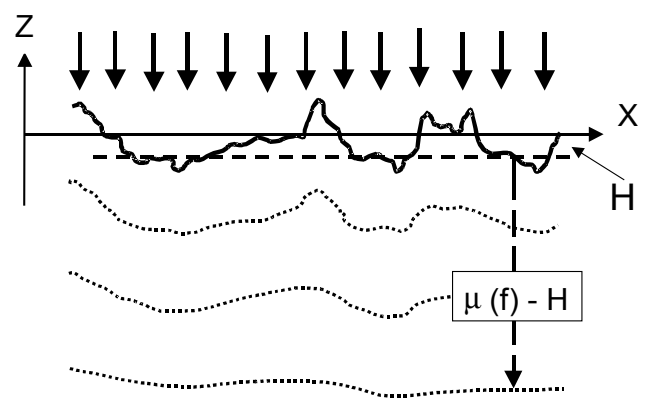

Fig. 3. Isotherms behind a rough surface absorber. They become smoother with increasing distance from the surface. The equivalent smooth surface is shifted by the offset $H$ with respect of the mean surface $z=0$.

As sketched in Fig.3. $\mathrm{k}_{\min }$ is the reciprocal maximum wavelength of the profile and prefactor $\mathrm{C}(\varsigma)$ is related to Riemann's Zeta-function $\mathrm{Z}(\varsigma)$ by 


$$
C(\varsigma)=\pi \cdot \frac{Z(2 \varsigma)}{Z(2 \varsigma+1)}
$$

Now we turn our interest to thermal waves propagating from rough surfaces. As mentioned above the heat diffusion in the vicinity of a rough surface is obstructed due to roughness. In distinction to [7] we have to replace the isotherms by the phase fronts of thermal waves.

Three scales are essential for non-stationary heat transportation across the rough surface: profile numbers $s$ and $L_{\max }$ and thermal diffusion length $\mu(f)$. It is plausible that the distortion of thermal waves becomes weaker for smoother surfaces (small $s$ and large $L_{\max }$ ). Low frequency thermal waves with long diffusion lengths average over small and short-ranging surface undulations which are evaluated by the high frequency wing of $\mathrm{g}(\mathrm{k})$. Short thermal waves are of course much more sensitive to surface undulations. The scale of smoothing out thermally the roughness is of the order of $\mu(f)$. That is why high frequency thermal waves become more strongly distorted by the roughness than low frequency ones. The longer the thermal diffusion length is (corresponding to decreased modulation frequencies $\mathrm{f}$ ), the weaker is the influence of high spatial frequency content on thermal wave propagation. We suppose that $\mu(f)$ estimates the lower cut-off frequency $\mathrm{kmin}$ of PSD which is responsible for thermal wave distortion.

In the limit $\mathrm{f} \rightarrow 0$ the offset $\mathrm{H}$ is given by equ.(5). This result is also reasonable for sufficiently long diffusion lengths $\left(\mu \geq L_{\max }\right)$. For shorter diffusion lengths we resort to the general definition of offset $\mathrm{H}$ given in [7]. With approximation $\mathrm{k}_{\max } \rightarrow \infty$ we get

$$
H=-\int_{k_{\min }}^{k_{\max }}\left|Z^{2}(k)\right| \cdot k \cdot d k=-\int_{1 / \mu(f)}^{\infty}|Z(k)|^{2} \cdot k \cdot d k
$$

For self-affine profiles with $\varsigma>0.5$ and lower bandwidth limit $k_{\min }=2 \pi / L_{\max }$ the shortening of the thermal propagation length in the near-surface region becomes

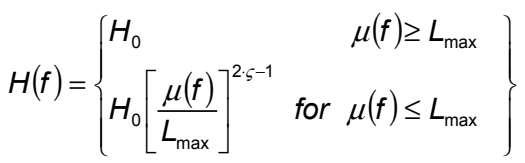

with $H_{0}=C(\varsigma) \cdot \frac{s^{2}}{L_{\max }}$

\section{Model calculations}

Due to the roughness the effective thermal diffusion length $\mu^{\prime}$ in the subsurface region is shorter than the bulk value $\mu$

$$
\mu^{\prime}(f)=\mu(f) \cdot r(f)
$$

with reduction factor $r(f)=(1-H / \mu(f))$. Of course this equation is only reasonable for $\mu>2 \cdot R p$ (or: $\mu>2 \cdot s$ ). In the subsurface range we may conclude from equ.(8)

$$
\alpha^{\prime}(f)=\alpha(f) \cdot r^{2}(f) \text { and } \quad \varepsilon^{\prime}(f)=\varepsilon(f) \cdot r^{2}(f)
$$

There is ambiguity of the definition of 'k' and $\rho c^{\prime}$ which can be overcome only by additional assumptions about the model and by comparison with experiments. 
Combining equ.(4) with (7) - (10) allows for the calculation of frequency dependent signals. Such calculations have been performed to demonstrate that the model is suited for describing correctly the observed experimental results. An example representing rough steel is shown in Fig. 4. The calculated curves illustrate the influence of rms roughness $s$ at fixed $\zeta$ and $L_{\max }$. For equal roughness exponents and correlation lengths but increased rms roughness the signals deviate more from the smooth-sample signals, demonstrating the decisive influence of surface texture on high frequency photothermal measurements.

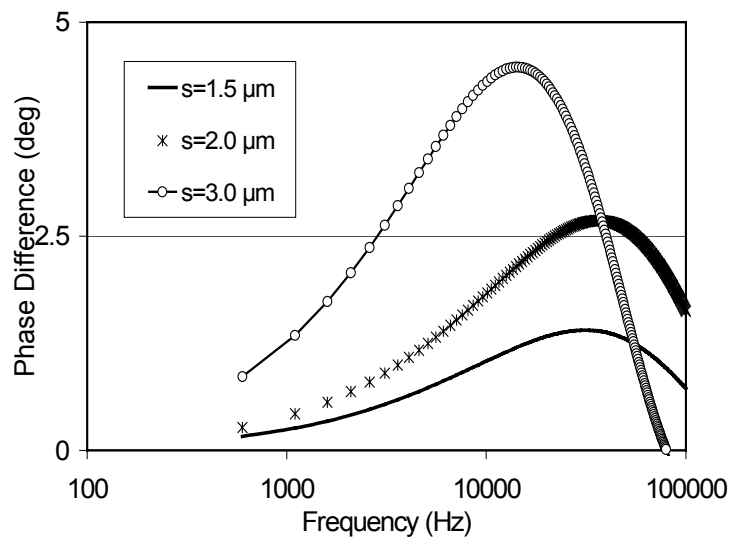

Fig. 4. Calculated frequency scans from self-affine rough samples. Fixed parameters are $\zeta=0.65$ and $L_{\max }=30 \mu \mathrm{m}$

\section{Experimental validation}

We compared radiometric measurements from roughened steel specimens with numerical calculations based on equ. (4) and (7)-(10). Three steel cubes (labeled by R1, $\mathrm{R} 2, \mathrm{R} 3$ ) of about $2 \mathrm{~cm} 3$ volume and different surface finish were studied. Surface texture parameters $\mathrm{Rp}, \mathrm{Rq}$ and $\mathrm{RSm}$ were estimated profilometrically. We performed conventional photothermal radiometric measurements [8] using a frequency doubled $5 \mathrm{~W} \mathrm{Nd}$ :YAG laser as the heat source. The infrared radiation was picked up by a LN2 cooled MCT detector. To give an example, the normalized signals from R2 are presented in Fig.5. A satisfactory agreement between the calculations and the experiment could be achieved for appropriately guessed model parameters $\mathrm{d}, \mathrm{s}, \mathrm{L}_{\max }$ and $\zeta$. The fit results are summarized in Tab1. We obtained a good agreement between peak to valley distance $2 \cdot R p$ and effective layer thickness d. Also, the profilometrically estimated RSm and the fitted $L_{\max }$ correspond to each other. The fitted rms roughness $s$ follows qualitatively the expected behavior. In spite of the rough and indirect estimation of $s, d$ and $L_{\max }$ the correspondence to the profile numbers $\mathrm{Rp}, \mathrm{Rq}$ and $\mathrm{RSm}$ is reasonable.

Table 1. Steel samples - Comparison between profilometrically estimated surface texture parameters $R p, R q$ and $R S m$ and photothermally fitted parameters $d, s, L_{\max }$ (distances in $\mu \mathrm{m})$ and roughness exponents $\zeta$ (Sample $R 1$ is used as reference).

\begin{tabular}{|c|ccc|cccc|}
\hline Sample & $\mathbf{R p}$ & $\mathbf{R q}$ & $\mathbf{R S m}$ & $\mathbf{d}$ & $\mathbf{s}$ & $\mathbf{L}_{\max }$ & $\zeta$ \\
\hline Steel R1 & 0.87 & 0.11 & 32 & - & - & - & - \\
Steel R2 & 3.06 & 0.37 & 29 & $4.5 \ldots 6.0$ & $1.5 . .2 .0$ & 35 & $0.60 \pm 0.05$ \\
Steel R3 & 5.19 & 0.79 & 37 & 7.5 & 2.5 & 25 & $0.55 \pm 0.02$ \\
\hline
\end{tabular}



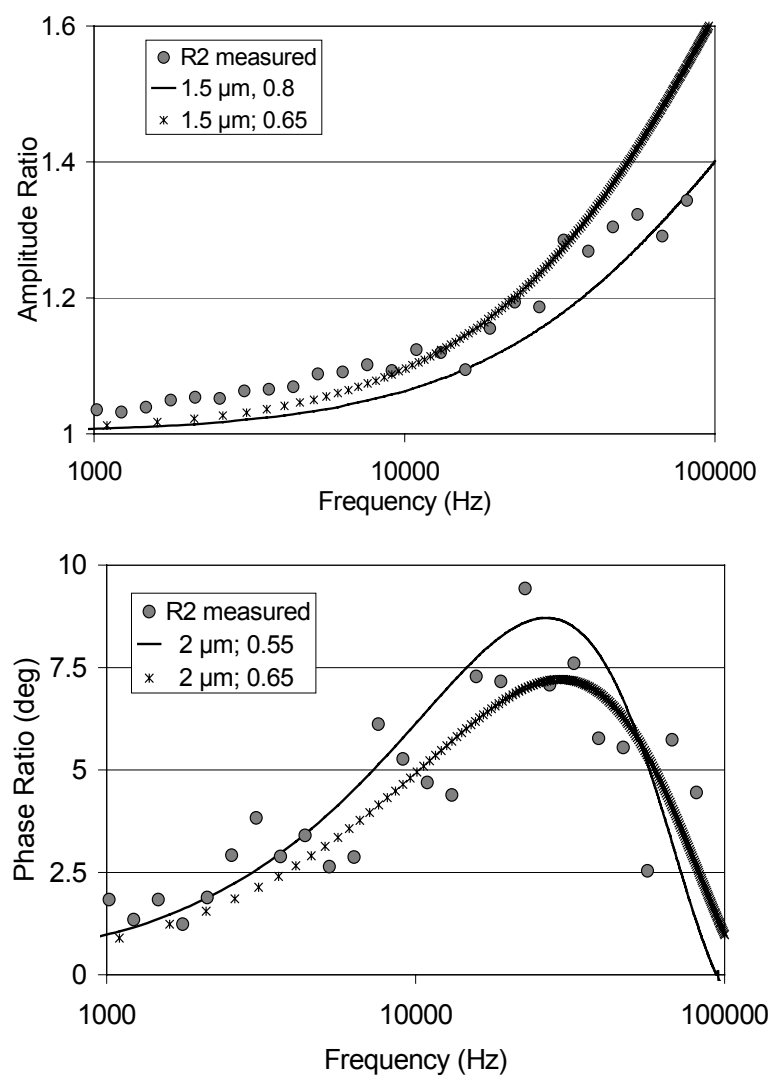

Fig. 5. Comparison between theory and experiment for steel sample R2. Fit parameters are shown in the legend. $L_{\max }=35 \mu \mathrm{m}$.

\section{Summary and discussion}

Photothermal frequency scans are distorted by surface roughness. This effect has to be taken into account for quantitative interpretation of photothermal measurements in the high frequency range (for instance: subsurface depth profiling or microstructure inspection). We combined the existing effective layer model $[2,3,4]$ with the effect of obstruction of thermal diffusion due to roughness, and derived an adequate expression for photothermal signals. The essential result of our model is the roughness induced dispersion of thermal waves caused by the frequency dependent shortening of the thermal diffusion length.

The performance of the proposed model was successfully demonstrated by comparing calculations with radiometric measurements from rough steel specimens. A reasonable agreement between measured and fitted roughness parameters was obtained.

The value of the proposed model consists in the correct description of photothermal frequency scans. If samples under study fall within the scope of the model, the reasonable order of magnitude of surface texture parameters can be derived. However, any unambiguous and quantitatively exact characterization of the surface roughness is out of the frame of the effective layer model. 


\section{REFERENCES}

[1] H.G. WALTHER, M.C. LARCIPRETE, P. MAYR, S. PAOLONI, B.S CHMITZ, Analytical Sciences, Vol. 17 Special Issue 2001, p 428

[2] L. NICOLAIDES, A. MANDELIS, C.J. BEINGESSNER, Analytical Sciences, Vol. 17 Special Issue 2001, p 383

[3] P.M. PATEL, D.A. ALMOND, H. REITER, $4^{\text {th }}$ Int.Top.Meeting on Photoacoustic Thermal and Related Sciences, Quebec 1985, WB 9.1; B.K. BEIN, S. KRUEGER, J. PELZL, $4^{\text {th }}$ Int.Top.Meeting on Photoacoustic, Thermal and Related Sciences, Quebec 1985, WA 4.1

[4] H.G. WALTHER, J.Appl.Phys. 89 (2001) p 2939

[5] D. VANDEMBROUCQ, C.BOCCARA, Progress in Natural Science, Suppl. Vol. 6 (1996), cp 313

[6] R. OSIANDER, P.KORPIUN, , Progress in Natural Science, Suppl. Vol.6, (1996), p 653-656

[7] D. VANDEMBROUCQ, S.ROUX, Phys.Rev. E 55(1997), p 6171

[8] T.T.N. LAN, U. SEIDEL, H.G. WALTHER, G.GOCH, B. SCHMITZ, J.Appl. Phys. 78, (1995), p 4108 